\title{
Three-Compton Telescope: Theory, Simulations, and Performance
}

\author{
R. A. Kroeger, W. N. Johnson, J. D. Kurfess, B. F. Phlips, E. A. Wulf
}

\begin{abstract}
The advent of highly segmented gamma ray detectors with good energy resolution has made a new class of gamma ray detectors possible. These instruments record the positions and energies of each individual gamma-ray interaction with high precision. Analysis of the individual interactions can provide energy and directional information, even for events with only partial energy deposition. Advantages over traditional gamma ray detectors include enhanced efficiency, background rejection, gamma ray imaging, and sensitivity to polarization. Consider those gamma rays that interact three or more times in the detector. The energy of the gamma ray that initiated one of these events is uniquely determined by measuring the energies of the first two interactions, and the scatter angle of the second interaction. The precision of this measurement is limited by the energy and position resolution of the detector, but also from Doppler broadening that results from the scattering off of bound electrons in the detector. It is also essential to correctly sequence the first three interactions. The importance of Doppler broadening is greater in higher Z-materials, thus silicon becomes a good choice for the detector material. We discuss performance and simulations of the multiple Compton telescope.
\end{abstract}

\section{INTRODUCTION}

$\mathrm{T}$ RADITIONAL gamma ray detectors must completely absorb the energy of a gamma ray in order to determine its energy. A gamma ray may interact several times within the detector before either being absorbed in a photo-electric interaction, or possibly escaping the detector. Those gamma rays that escape lead to features such as a Compton shelf below the photo-peak energy, and a loss of photo-peak efficiency. An alternative detection technique is to carefully measure the positions and energy loss of each interaction. A subset of these events will consist of three or more

This work was supported in part by the Office of Naval Research, the Defence Threat Reduction Agency, and the National Aeronautics and Space Administration.

R. A. Kroeger is with the Naval Research Laboratory, Washington, DC 20375 USA (telephone: 202-404-7878, e-mail: kroeger@nrl.navy.mil).

W. N. Johnson is with the Naval Research Laboratory, Washington DC 20375 (telephone: 202-767-6817, e-mail: johnson@gamma.nrl.navy.mil).

J. D. Kurfess is with the Naval Research Laboratory, Washington DC 20375 USA (telephone: 202-767-3165, e-mail: kurfess (a)gamma.nrl.navy.mil).

B. F. Phlips is with the Naval Research Laboratory, Washington DC 20375 USA (telephone: 202-767-3572, e-mail: phlips (a)gamma.nrl.navy.mil).

E. A. Wulf is with the Naval Research Laboratory, Washington DC 20375 USA (telephone: 202-404-1475, e-mail: wulf @gamma.nrl.navy.mil). interactions, beginning with two or more Compton events, followed by either a photoelectric event or escaping the detector. The energy of a gamma ray is uniquely determined by the energy loss of the first two interactions, and the scatter angle of the second interaction [1]. We give this process the generic name of "Three-Compton," referring to the three interactions and the application of the Compton formula to estimate the energy. The energy of the incident gamma ray, $E_{0}$, is given by the formula,

$$
E_{0}=\Delta E_{1}+\frac{\Delta E_{2}+\sqrt{\Delta E_{2}^{2}+4 \Delta E_{2} m_{e} c^{2} /\left(1-\cos \theta_{2}\right)}}{2}
$$

where $\Delta E_{1}$ and $\Delta E_{2}$ are the energy loss of the first and second interaction, and $\theta_{2}$ is the Compton scattering angle of the second interaction. Knowledge of the position of first, second, and third interactions is required to determine $\theta_{2}$, however knowledge of the third energy loss is not required.

Three-Compton has several unique properties: (1) efficient detectors in the $\mathrm{MeV}$ region are possible using low- $Z$ elements such as silicon, (2) the Compton shelf below (or above) the photo-peak is small, especially true for energies above a few $100 \mathrm{keV}$ where the process works best, (3) total energy absorption is not required, and (4) it naturally provides for an imaging a gamma ray detector.

We shall not discuss the imaging capability of a Compton telescope here. It is sufficient to note that with the measured positions of the first and second interactions, and with the measured total energy, the three-Compton detector is an example of a Compton telescope. The first two positions determine the direction of the first scattered gamma ray, and the energy losses provide the scatter angle. The direction of the incoming gamma ray is therefore restricted to a cone, and the superposition of many such event-cones can be used to generate an image. Many examples of Compton telescopes are in the literature [e.g. 2].

The efficiency of a three-Compton telescope can be quite high because full-energy detection does not require total absorption. Consequently, the energy of events that would otherwise fall in the Compton shelf of a traditional detector are properly measured with this method. Naturally, the threeCompton telescope must be large enough that the probability of having three interactions is significant.

The determination of the correct order of the interactions for each event is a key factor in efficiency. We presume that direct measurement of event order through fast timing or other techniques is not practical, thus the interaction order 
must be deduced from the observed energies and positions. There are six possible sequences of interactions for events with three interactions. Of these, only the correct sequence will provide the correct incoming energy using Equation 1, and the correct event-cone for imaging. The other five sequences would provide an erroneous energy and eventcone. Improper sequencing is both a loss of efficiency, and a source of background. In general, there are $n$ ! sequences for events with $n$ interactions. We shall discuss a simple algorithm to determine the interaction sequence with reasonable to good efficiency.

The three-Compton process does not require a high- $Z$ detector, i.e. high stopping power. In fact, the method requires at least three interactions, thus high- $Z$ materials such as $\mathrm{CdZnTe}$ or NaI would not be good choices for energies below around $400 \mathrm{keV}$, where the probability of photoelectric absorption in the first or second is starting to be either significant or dominant.

\section{Simulations}

The estimated energy given by Equation 1 is based on the well-known Compton scattering formula. However, this is only strictly valid for scattering from cold free electrons. In reality, electrons are bound in atoms. The electron momentum affects the scatter angle in a process called Doppler broadening. Doppler broadening contributes an uncertainty in the energy estimate [3].

A simple IDL ${ }^{\circledR}$ code was developed to perform gamma-ray transport in a Monte-Carlo simulation of simple instrument configurations. The program provides positions and energies of interactions for a simulated beam of gamma rays. It includes the physical processes of gamma-ray cross-sections, ranges, pair production, coherent scattering, Compton scattering and Doppler broadening [4], [5]. It does not propagate recoil electrons, which could become important in thin or low density detectors, or at high energies. The physics included is sufficient to demonstrate the efficiency, interaction ordering, and energy resolution that can be expected in a realistic instrument.

\section{A. Detector resolution}

The energy and spatial resolution of the detectors has a significant impact on the accuracy of the energy reconstruction. Figure 1 shows a reconstruction from simulated data. The two curves are produced from the same data, one with perfect knowledge of energy loss and positions, and the other with the data degraded to represent a realistic detector. Detector energy resolution is presumed to follow gaussian distribution about the true energy, and position resolution is divided into voxels (boxes) with sharp boundaries. The reconstructed energy has a sharp nongaussian central peak and broad wings, which result from the Doppler broadening in the simulation. We note that the wings of the spectrum representing the realistic detector are reduced if we select only those data where the distance between interactions is a greater than some minimum distance. The appropriate choice of this minimum distance reduces the wings slightly, with little affect on the amplitude of the peak. Setting the minimum distance too large will cut into the efficiency, though eventually the shape of the distribution will resemble that produced by the perfect data.

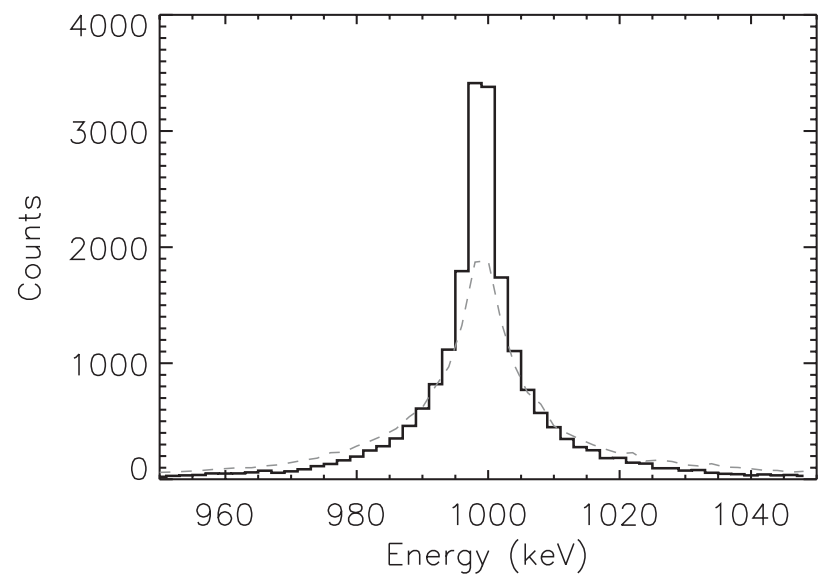

Figure 1: Reconstructed energy spectrum for a $1 \mathrm{MeV}$ gamma ray beam in silicon. The solid line represents an ideal silicon detector with perfect energy and position resolution. The light dashed curve represents a detector with 2 keV FWHM energy resolution and $2 \mathrm{~mm}$ spatial resolution.

\section{B. Sequencing}

Practical 3-Compton detectors are not capable of determining the order of the interactions in each event. This was done in COMPTEL by fast timing between detectors 1.5 meters apart [2]. The 3-Compton telescope is presumed to bring the detectors into close proximity in order to increase the efficiency, thus light propagation times are much shorter. Further, the fast timing possible in COMPTEL would require far more power and/or significant advances in detector technology with the segmented semiconductors considered for this application. Thus, the event order must be determined by observing the pattern and amplitude of energy losses within the detector.

Consider those events with exactly three interactions. There are six possible permutations in which to order these interactions. The sequence with the correct order will be fully consistent with Compton kinematics. A simple way to test a sequence is to apply Equation 1 to find an energy, $E_{0}$, for that sequence. Two tests can be applied: (1) is the first measured energy loss, $\Delta E_{1}$, a valid Compton scatter value, given an incident energy of $E_{0}$ ? An example of a rejected sequence might reconstruct to $1 \mathrm{MeV}$, with an initial energy loss of $0.90 \mathrm{MeV}$, and a scattered gamma ray of $0.10 \mathrm{MeV}$. Clearly this is than the maximum energy loss of $0.80 \mathrm{keV}$ from a 180-degree Compton scatter, and therefore is not physical. (2) is the third energy loss consistent with either a Compton scattering event, or a photoelectric event? The same argument applies, with the exception that there is also a possibility that the last event is a photoelectric absorption.

Tie-breakers can be resolved by application of a score, or Figure of Merit (FOM) that is computed for each valid 
sequence. Possible FOM terms are derived from probabilities of the chain of events in the sequence. These may include the differential cross-section for each scatter angle, thus rare scatter angles would be given lower weight. The FOM may also consider other factors such as the range probabilities for each gamma ray in the sequence, or the range of the first or last interaction to the entry or exit points of the detector.

Giving preference to those sequences that end with a photoelectric event provides a third tiebreaker. The photoelectric cross-section for the final interaction may well be smaller than the corresponding Compton cross section. However, the probability of an interaction landing in the narrow energy window that defines a photoelectric ending is relatively small. Thus, this tends to be a powerful discriminator.

The effectiveness of sequencing algorithms was studied using the simulated data from a large silicon detector. The algorithm was able to identify the correct sequence $40 \%$, $47 \%$, and $80 \%$ of the time at 185,414 , and $2615 \mathrm{keV}$ respectively for three interactions.

We note that the range probability FOM does not help in the three-interaction example. Improper sequences often reconstruct to higher energies, and higher energy gamma rays have longer ranges. Thus the range statistic prefers to select higher-energy solutions. The range statistic does improve sequencing slightly with four or more interactions. The best reconstruction strategy for a given instrument, energy, or other variables lends itself well to Monte Carlo analysis.

Another powerful term is added to the FOM with four or more interactions. Consider four interactions: an incident energy is determined using Equation 1 for the first three interactions, and an independent energy is calculated using the last three interactions (the energy $E_{1}$ is determined from the last three interactions through Equation 1 in much the same manner as $E_{0}$ is determined from the first three interactions. $E_{0}$ is then found by adding the first energy loss, $\Delta E_{1}$ ). These two determinations of $E_{1}$ should be the same within a reasonable error window. There are 24 possible sequences for four interactions.

Our algorithm was able to identify the correct sequence $45 \%, 64 \%$, and $87 \%$ of the time at 185,414 , and $2615 \mathrm{keV}$ respectively.

This approach easily generalizes to 5 or more interactions. Each sequential set of three interactions is an independent estimate of energy, and they must all be self-consistent. Each sequence is assigned a FOM component based on the difference between each estimator of energy, and the sequence with the highest over-all FOM is selected as "best." Event ordering is computationally limited above about six interactions. There are 720 possible sequences with six interactions, each of which must be evaluated to determine the best. Presently, we reject events with seven or more interactions until a more sophisticated algorithm can be developed to limit the search.

\section{Realistic Detectors}

Energy resolution and position resolution degrade the accuracy of both the reconstructed energy and the sequencing efficiency. This is shown in Figure 2 for various values of detector position resolution. A detector energy resolution of $2 \mathrm{keV}$ FWHM was used. It is evident that position resolution has the most effect on reconstructed energy resolution at higher energies. This also implies that higher density detectors with correspondingly shorter gamma ray ranges may also be strongly affected. We note that the silicon detector in this simulation had an average density of 0.8 $\mathrm{g} / \mathrm{cm}^{3}$ due to the assumed gaps between the detectors.

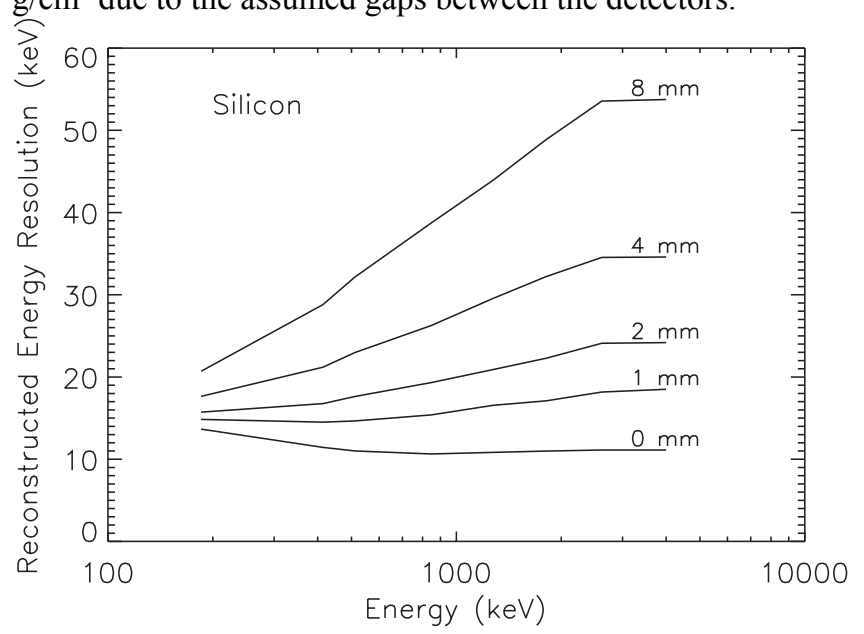

Figure 2: Reconstructed energy resolution vs. incident energy for various detector voxel sized (position resolution). The detector energy resolution is 2 keV FWHM.

\section{EFFICIENCY}

The efficiency of 3-Compton telescope is limited by a combination of: a) size of the detector, b) producing three or more detectable interactions, c) properly sequencing the event order, d) passive materials within the detection volume where key interactions may be lost, and e) no more than six interactions with our present computer power and algorithm. Figure 3 shows the expected efficiency after pulling all of these factors together for a $1 \mathrm{~m}^{2}$ area detector, with a total thickness of $43 \mathrm{~g} / \mathrm{cm}^{2}$. A detection threshold of $10 \mathrm{keV}$ was used, thus coherent scatters and very small angle Compton scatter events are not detected. Events with undetected interactions constitute one component of lost efficiency. The low threshold is most significant for lower energy gamma rays where coherent and low energy-loss Compton scattering is more likely. 


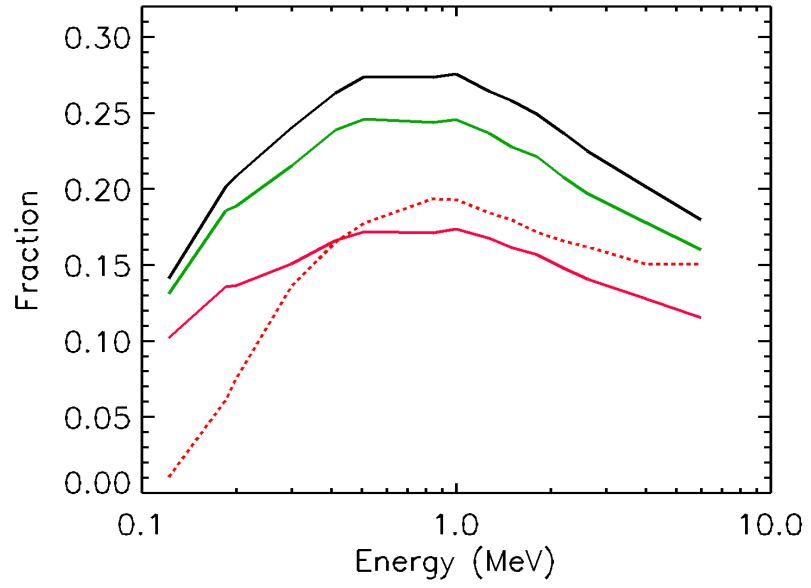

Figure 3: Efficiency of a 3-Compton telescope vs. energy. The three solid curves are for a silicon detector, the dashed curve is for germanium. The upper curve shows the fraction efficiency for an ideal silicon detector with perfect position and energy resolution. The next curve below this shows the same detector with $3 \mathrm{keV}$ FWHM energy resolution and $1 \mathrm{~mm}$ voxel resolution. The lower solid curve represents this detector with $10 \%$ passive materials. The dashed curve is for a similar sized germanium detector with realistic resolution and $10 \%$ passive materials.

There is potential improvement in the efficiencies shown here by utilizing those events with 7 or more interactions. A potential gain of around $50 \%$ is possible for the silicon curves at high energies by this improvement alone. It may also be possible to further improve the sequence selection algorithm. Presently we only count valid sequences to determine efficiency, however a fair number of five and six interaction sequences have the first three and most critical interactions sequenced correctly. What happens after the third interaction need not invalidate a sequence. Efficiency of the silicon 3-Compton telescope could also be improved by considering a hybrid instrument that includes thin layers of a high-Z detector such as CdZnTe. The CdZnTe would increase the probability of absorbing low energy photons toward the end of the sequence, and reduce the total number of interactions in the sequence.

\section{CONCLUSIONS}

The simulated three-Compton efficiency shown in Figure 3 is well over an order of magnitude higher than the slightly larger COMPTEL instrument on the Compton Gamma Ray Observatory. Efficiency also extends to much lower energies than does COMPTEL. The reasons for improvement are simple: more compact geometry, and ability to utilize a much larger fraction of the events, including those that are not total absorption events, and ascribing no significance to where in the detector that the first interaction occurs. COMPTEL required that the first interaction be in the upper detectors. The lower energy performance is largely a matter of using low noise semiconducting detectors to initially scatter the gamma ray vs. liquid scintillators in COMPTEL, and that
COMPTEL primarily relied on scatters of less than roughly 30 degrees.

The sequencing algorithm may be improved slightly. The optimum FOM may be determined through Monte Carlo simulations. Improvements are possible by fine-tuning the FOM as a function of energy, or by prior knowledge about the source.

Applications that require a large, imaging field of view are likely to benefit from the three-Compton approach. These include astrophysics where long integration times and fullsky coverage are essential to capture faint and transient events such as supernovae explosions. This may also be applicable in survey applications where imaging may help isolate radioactive hot spots, and reduce susceptibility to ambient backgrounds.

\section{REFERENCES}

[1] J. D. Kurfess, W. N. Johnson, R. A. Kroeger and B. F. Phlips, "Considerations for the next Compton telescope mission", AIP Conf. Proc. 510, pp. 789-793, 2000.

[2] V. Scholnfelder, "COMPTEL," ApJS, Vol. 86, 657, 1993.

[3] Y.F. Du, Z. He, G.F. Knoll, D.K. Wehe, W. Li, "Evaluation of a Compton scattering camera using 3-D position sensitive CdZnTe detectors," Proc. SPIE, vol. 3768, pp. 228, 1999.

[4] Y. Namito, S.Ban, H. Hirayama, "Implementation of the Doppler broadening of a Comtpon-scattered photon into the EGS4 code," Nucl. Instr. And Meth., A 349, 489-494, 1994.

[5] F. Biggs, L.B. Mendelsohn, J.B. Man, "Hartree-Fock Compton profiles for the elements," Atomic Data and Nucl. Data Tables, 16, 201-309, 1975. 